\title{
Révision de la LPMéd: tout est bien qui finit bien?
}

\author{
Jürg Schlupa ${ }^{a}$ Christoph Hänggeli ${ }^{b}$ \\ a Dr, président de la FMH \\ b Avocat, directeur de I'ISFM
}
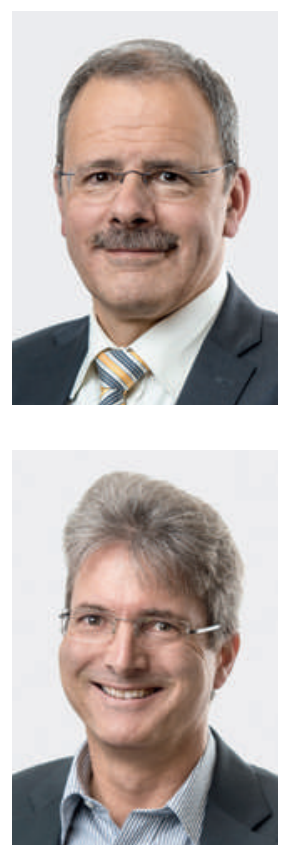

Le 20 mars 2015, soit un peu moins de quatre ans après l'ouverture de la procédure de consultation, les chambres fédérales ont définitivement approuvé le projet de révision de la loi sur les professions médicales universitaires (LPMéd). Le temps est venu de dresser un bilan et de se demander si le temps investi et le travail de sensibilisation accompagné d'un nombre impressionnant de prises de position, de séances, d'auditions et de communiqués de presse ont été payants. La réponse est oui, sans aucun doute.

A l'issue d'un long processus législatif et avec le soutien de la Société suisse des médecins-dentistes (SSO), Chirosuisse, pharmaSuisse et la Société des vétérinaires suisses (SVS), l'ISFM et la FMH sont parvenus à faire inscrire dans la loi deux aspects importants de la protection des patients. Toutes les personnes qui exercent une profession médicale doivent désormais remplir les conditions suivantes:

- figurer dans le registre des professions médicales, inscription qui garantit notamment la vérification officielle des diplômes étrangers;

- justifier les connaissances linguistiques nécessaires à l'exercice de leur profession.

\section{Un signe fort en faveur d'une plus grande protection du patient: tous les diplômes de médecin étrangers feront désormais l'objet d'une vérification et d'un enregistrement officiels.}

Limitées jusqu'à présent aux seuls indépendants, ces conditions s'appliquent maintenant à tous les médecins salariés. Cette extension du domaine d'application de la LPMéd représente une avancée notoire. L'obligation de figurer dans le registre va profiter non seulement aux patients mais aussi aux hôpitaux: lors du recrutement de personnel étranger, la vérification des diplômes sera désormais entièrement du ressort de la Commission des professions médicales, préservant les hôpitaux d'une tâche complexe. L'inscription dans le registre constitue par ailleurs un gage de fiabilité, tant pour le public que pour les employeurs, non seulement

\section{Il appartient au Conseil fédéral de trouver une} solution efficace et peu bureaucratique pour la mise en œuvre de la compétence linguistique par voie d'ordonnance.

en ce qui concerne les diplômes mais aussi les compétences linguistiques. Finis les médecins présumés qui exercent sans diplôme ou avec un faux diplôme, et passent librement d'un canton à l'autre sans risquer d'être démasqués. Fini aussi le manque de connaissances suffisantes d'une des langues nationales pour se faire comprendre des patients.

Est-ce pour autant le happy end espéré? Pas encore. Telle une hirondelle qui ne ferait pas le printemps, ce texte de loi ne peut pas à lui seul, et comme par un coup de baguette magique, corriger tous les dysfonctionnements existants. $\mathrm{Si}$, comme chacun sait, le diable se cache dans les détails, les dispositions d'application sont édictées par voie d'ordonnance et relèvent de la Confédération. Après les débats très nourris tant au Conseil des Etats qu'au Conseil national, on peut espérer que le Conseil fédéral ne réduira pas la compétence linguistique à un tigre de papier et saura aussi éviter le piège d'une bureaucratisation excessive. On pourrait par exemple imaginer que tous les détenteurs d'un diplôme de médecin provenant de régions linguistiques francophones, germanophones ou italophones n'aient pas à fournir de preuve supplémentaire de leur compétence linguistique. Pour les quelques pour cents restants, il faudra en revanche exiger une attestation de compétence linguistique vérifiable.

Si le processus d'élaboration du droit d'ordonnance débouche sur un résultat tout aussi fructueux que celui des quatre années de la révision de la loi, nous pourrons en 2016 nous réjouir d'une étape importante franchie en politique de la santé! 